\title{
ENFERMEDADES METABÓLICAS Y SU IMPACTO EN LA PRODUCTIVIDAD DEL GANADO LECHERO.
}

Alejandro Saborío-Montero ${ }^{1}$

\section{INTRODUCCIÓN}

En este artículo se resumen los hallazgos obtenidos de una serie de proyectos de investigación sobre enfermedades metabólicas en ganado lechero. Estos proyectos fueron adscritos a la Vicerrectoría de Investigación de la Universidad de Costa Rica en el periodo comprendido entre el año 2011 y 2015, y se desarrollaron de manera conjunta entre el Centro de Investigaciones en Nutrición Animal y la Escuela de Zootecnia.

Las enfermedades metabólicas son un grupo de padecimientos que afectan el metabolismo del animal y que usualmente ocurren en el periodo de transición, este periodo comprende desde 30 días antes hasta 30 días después del parto. Estas enfermedades disminuyen el desempeño productivo y reproductivo de los animales, se asocian a la presentación de otros padecimientos metabólicos e infecciosos, pueden causar la muerte en animales no atendidos apropiada y oportunamente, y reducen la rentabilidad de los sistemas productivos en los cuales ocurren (NRC, 2001).

Una de estas enfermedades es la cetosis, la cual es causada por cambios abruptos en el metabolismo energético del animal, ésta generalmente se presenta cuando el animal tiene un estado energético negativo, inducido por la disminución del consumo de alimento, el aumento en los requerimientos nutricionales para la producción de calostro y leche al inicio de la lactancia o ambos (Duffield, 2000).

Este estado energético negativo genera lipólisis de triglicéridos en los adipocitos, que han sido acumulados como grasa subcutánea en el animal. Uno de los productos de esta lipólisis son los ácidos grasos no esterificados, los cuales viajan por la sangre hasta el

\footnotetext{
1 Universidad de Costa Rica, Escuela de Zootecnia y Centro de Investigaciones en Nutrición Animal. San José, Costa Rica. Autor para correspondencia: alejandro.saboriomontero@ucr.ac.cr 
Saborío-Montero. Enfermedades Metabólicas y su impacto en la productividad del ganado lechero

hígado, son transformados a cuerpos cetónicos, principalmente a $\beta$-hidroxibutirato $(\beta \mathrm{HBA})$ y posteriormente liberados en sangre (Drackley, 1997).

Una elevada concentración de cuerpos cetónicos en sangre (1,4 a 2,9 mmol/L $\beta H B A)$ en ausencia de síntomas clínicos es definida como cetosis subclínica, la sintomatología clínica involucra: pérdida del apetito, disminución de la producción de leche, rápida pérdida de condición corporal, somnolencia y aliento con olor dulcete. Concentraciones sanguíneas superiores a 3,0 mmol/L de $\beta H B A$ son usualmente utilizadas como umbral de detección de cetosis clínica (Oetzel, 2007).

Durante el periparto también ocurre una disminución de calcio en sangre, generada por un aumento en la demanda feto-placentaria de este mineral, aunado a un incremento de los requerimientos nutricionales de calcio para la síntesis de calostro y leche. Esta disminución desencadena una respuesta fisiológica en el animal que consiste en un aumento en la concentración de la hormona paratiroidea, la cual incrementa la síntesis de $1,25(\mathrm{OH})_{2}$ vitamina D3 y la resorción ósea de calcio, lo que facilita la absorción de este mineral a nivel intestinal y la disponibilidad de calcio en sangre a partir de las reservas en hueso, respectivamente (Horst, 1986).

A pesar de esta respuesta fisiológica, algunos animales no son capaces de compensar este desbalance de calcio, lo que provoca otra enfermedad metabólica común denominada fiebre de leche o hipocalcemia.

La clasificación subclínica de esta enfermedad se asocia a una disminución de los niveles de calcio sanguíneo (1,4 a 2,0 mmol.l-1Ca). Por su parte, la clasificación clínica involucra síntomas que consisten en: movimientos atípicos, vaca caída, convulsiones y respuesta al tratamiento. Estos síntomas generalmente ocurren cuando la vaca tiene concentraciones sanguíneas de calcio inferiores a 1,4 mmo/L (Goff, 2006).

El objetivo de este manuscrito consiste en presentar los resultados más relevantes y recomendaciones aplicables a nivel de sistemas productivos lecheros para disminuir la incidencia de las enfermedades metabólicas analizadas. 
Nutrición Animal Tropical

\section{MATERIALES Y MÉTODOS}

Debido a que las razas, número de animales y hatos varió entre proyectos de investigación, no es posible describir estas variables. Sin embargo cada uno de los resultados aquí descritos posee una referencia asociada, en la cual se podrá encontrar una descripción detallada de éstas y otras variables de interés.

Para la detección de la concentración de $\beta H B A$ en sangre se muestrearon lo vasos sanguíneos coccígeos de cada animal con agujas de tamaño 21" x 1,5" y tubos VACUTTE de tapa roja. El equipo utilizado fue un medidor electroquímico de mano (Optium Xceed®, Abbott Diabetes Care $\AA^{\circledR}$ ) con alta sensibilidad y especificidad (Iwersen, Falkenberg, Voigtsberger, Furderung y Heuwieser, 2009). Se establecieron los umbrales mínimos para cetosis subclínica y clínica en 1,4 y 3,0 mmol/L $\beta H B A$ (Oetzel, 2007), respectivamente.

Se calificó la condición corporal de los animales en una escala de 1 a 5 puntos con incrementos de 0,25 puntos, mediante la metodología establecida por Ferguson, Galligan, y Thomsen (1994).

Para el diagnóstico de hipocalcemia se tomaron muestras de sangre de la misma manera y fueron trasladadas al Centro de Investigación en Nutrición Animal, donde fueron analizadas mediante espectrofotometría de absorción atómica (Perkin Elmer, A. Analist 800). Los umbrales por debajo de los cuales se diagnosticó hipocalcemia subclínica y clínica fueron 2,0 y 1,4 mmol/L (Goff, 2006), respectivamente.

Otro método de detección que fue tomado en cuenta para fiebre de leche fue la presentación de síntomas en los animales, detectados por los encargados de las lecherías comerciales durante el periparto y con respuesta positiva al tratamiento.

La información productiva, reproductiva y sanitaria de los hatos se registró a través del software VAMPP Bovino 3.0. (Noordhuizen y Buurman, 1984). Para el análisis de la información se utilizaron los paquetes estadísticos InfoStat 1.0. (Di Rienzo, Casanoves, Balzarini, González, Tablada y Robledo, 2011). e IBM SPSS Statistics®, Versión 22. 
Saborío-Montero. Enfermedades Metabólicas y su impacto en la productividad del ganado lechero

\section{RESULTADOS Y DISCUSIÓN}

\section{Cetosis}

En un estudio sobre cetosis en ganado bovino (Saborío-Montero y Sánchez, 2013), se evaluó la prevalencia de cetosis en un hato Jersey a los $8 \pm 3$ y $30 \pm 3$ días posparto en 117 y 114 vacas, respectivamente. En esta investigación se obtuvieron prevalencias de cetosis clínica de 0 y $3,51 \%$, en el mismo orden. El grado subclínico de la enfermedad se determinó en 4,27 y $9,65 \%$ de los animales para los periodos descritos. Estos resultados se consideran relativamente bajos en comparación con valores reportados en la literatura en otras latitudes (Duffield, 2000; Epperson, 2005).

La concentración promedio de $\beta \mathrm{HBA}$ determinada durante los periodos $8 \pm 3$ y $30 \pm 3$ días posparto fueron 0,66 y $0,91 \mathrm{mmol} / \mathrm{L}$, respectivamente. Tanto las concentraciones como la prevalencia de cetosis indicaron una tendencia a presentar cetosis tipo I (Disminución prolongada del consumo de alimento) sobre cetosis tipo II (Alta movilización de ácidos grasos a partir de reservas corporales).

La condición corporal de ese hato se calificó desde el secado hasta el final de la lactancia para generar la caracterización de la curva de condición corporal (Saborío-Montero y Sánchez, 2014) y para asociar los cambios en calificación de condición corporal (CCC) con la concentración de BHBA en sangre. En este estudio se determinó que a mayor CCC en la semana del parto, mayor pérdida de CCC hasta el punto más bajo (Nadir de CCC). Además se obtuvo que los animales que presentaron concentraciones de BHBA superiores o iguales a $1,4 \mathrm{mmol} / \mathrm{L}$ a los $30 \pm 3 \mathrm{~d}$ de lactancia, tuvieron una pérdida de CCC en la semana previa al parto significativamente $(p<0,05)$ mayor que los animales cuya concentración de $\beta$ HBA fue inferior a $1,4 \mathrm{mmol} / \mathrm{L}$ ( $-0,31$ vs $-0,09$ puntos de $C C C$ ).

Otro hallazgo importante relacionado a la incidencia de cetosis a los $30 \pm 3$ días posparto fue la relación de la enfermedad con el número de partos. Con cada parto, se incrementó $(p<0,01)$ la probabilidad de que una vaca sufriera cetosis en 1,38 veces (Figura 1$)$. 
Nutrición Animal Tropical

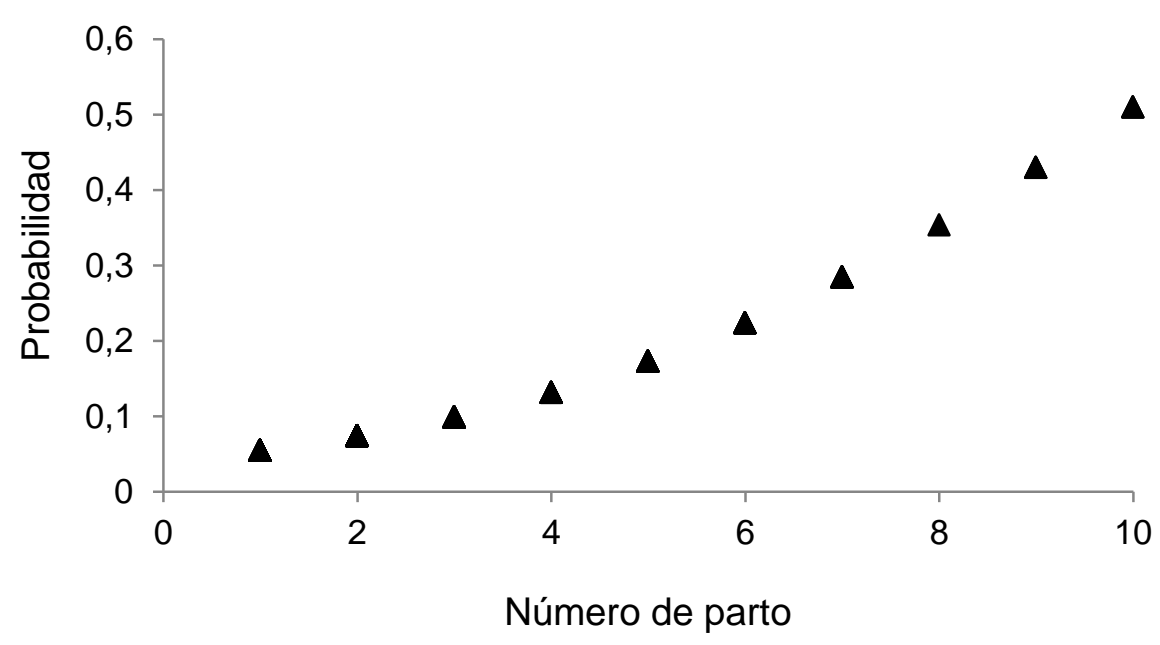

Figura 1. Razón de probabilidades de sufrir cetosis conforme aumenta el número de parto, según concentración de $\beta$ HBA promedio para animales sanos y enfermos

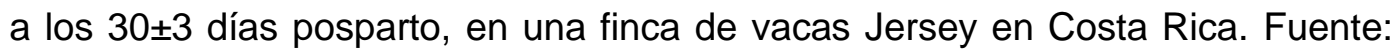
Saborío-Montero y Sánchez, 2013.

Además se determinó que los animales que presentaron concentraciones de $\beta H B A$ superiores o iguales a 1,4 mmol. $\mathrm{L}^{-1}$, tuvieron duración del periodo seco significativamente mayor $(p<0,05)$ en relación con los animales que tuvieron concentraciones de este cuerpo cetónico inferiores a este valor.

\section{Fiebre de leche}

Una investigación sobre la prevalencia de hipocalcemia en cuatro hatos Jersey en pastoreo en Costa Rica (Sánchez y Saborío-Montero, 2014a), determinó que durante el periparto, se presentaron concentraciones sanguíneas de calcio inferiores a 1,4 mmol/lL (Hipocalcemia clínica) en $25 \%$ o más de los animales a partir del cuarto parto. Además, los animales que presentaron valores de calcio entre 1,4 y $2.0 \mathrm{mmol} / \mathrm{L}$ (hipocalcemia subclínica) fueron más del $40 \%$ indiferentemente del número de parto (Figura 2). 
Saborío-Montero. Enfermedades Metabólicas y su impacto en la productividad del ganado lechero

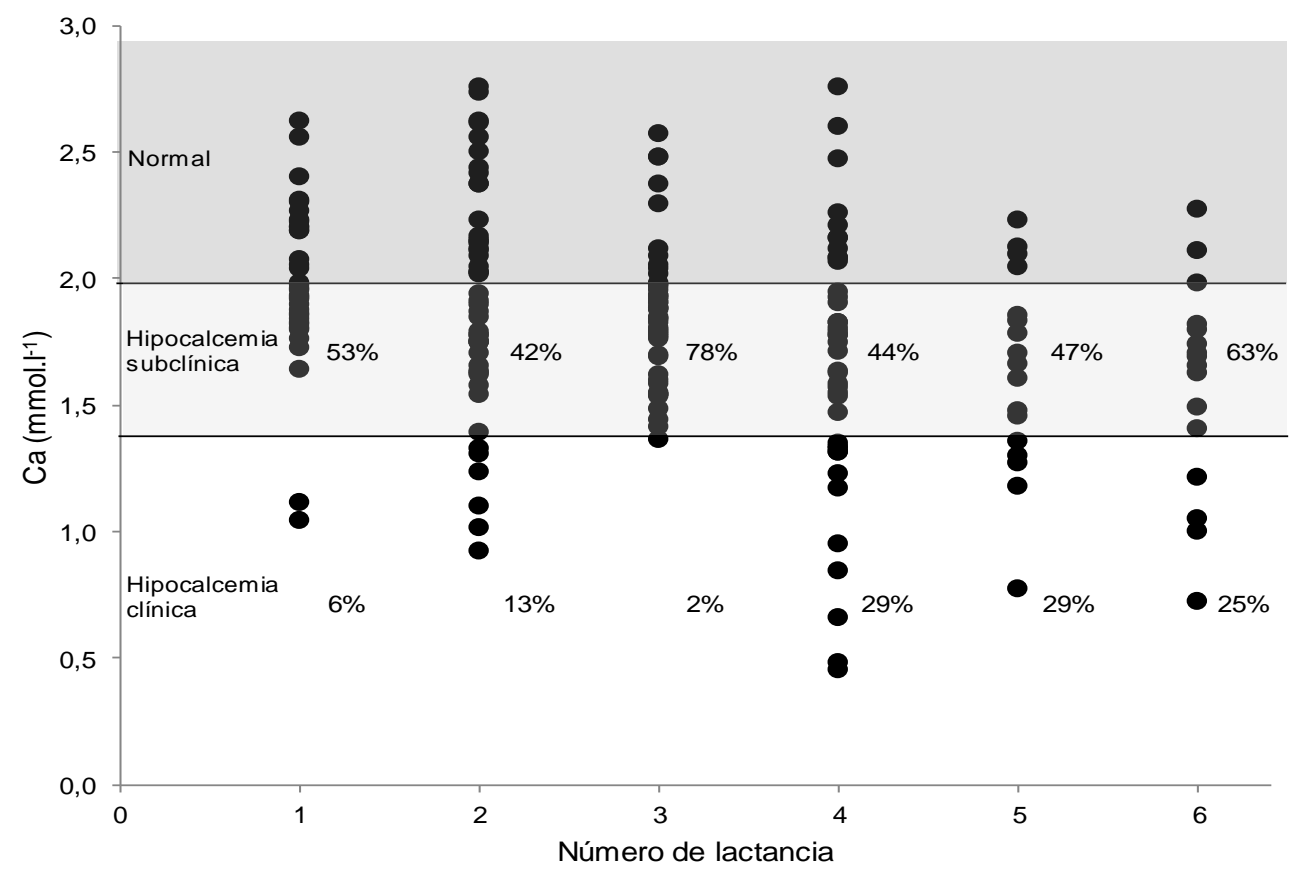

Figura 2. Concentración de calcio en el suero sanguíneo durante el periodo de $24 \mathrm{~h}$ previas y posteriores al parto en 4 hatos de la raza Jersey en pastoreo en Cartago, Costa Rica, diagramada según el número de lactancia y la prevalencia de hipocalcemia clínica, subclínica o normocalcemia. Fuente: Sánchez y Saborío-Montero, 2014a

Las concentraciones promedio de calcio en sangre de los animales en este periodo disminuyó conforme aumentó el número de parto y fue estadísticamente diferente $(p<0,01)$ entre animales de cuatro o más partos, en relación a animales de primer parto.

Otro estudio sobre hipocalcemia en vacas de las razas Holstein, Jersey y Guernsey (Sánchez y Saborío-Montero, 2014b) determinó que el efecto de disminución en la concentración de calcio durante el periparto se acentúo más en la raza Jersey, siendo esta la más propensa a desarrollar la enfermedad conforme aumenta el número de parto del animal.

En esta investigación se determinó una baja prevalencia (3,95\%) general de hipomagnesemia (deficiencia de magnesio). Las concentraciones de magnesio en los animales Jersey durante el periodo de estudio, fueron significativamente mayores 
Nutrición Animal Tropical

$(p<0,001)$ en relación a las razas Holstein y Guernsey. De estos hallazgos se concluye que los casos de hipocalcemia observados se deben a aspectos biológicos propios de la homeostasis del calcio en el animal y no a mecanismos en la fisiología de este mineral en los que interviene el magnesio.

\section{CONSIDERACIONES FINALES}

Bajo las condiciones propias de Costa Rica, la investigación relativa a las enfermedades metabólicas en ganado lechero es limitada. Algunas recomendaciones tendientes a la disminución del impacto de la cetosis y fiebre de leche sobre la productividad de estos sistemas productivos se resumen a continuación:

- Realizar un recambio de animales adecuado para eliminar vacas viejas problemáticas, las lecherías con vacas Jersey deben prestar especial atención.

- Medir y adecuar la condición corporal de los animales durante la lactancia para que las vacas paran con condición de 3,50-3,75.

- Evitar un periodo seco mayor de 60 días.

- Realizar un periodo de transición adecuado y con prácticas de alimentación según recomiende el Ingeniero Agrónomo Zootecnista o especialista en nutrición animal.

- Minimizar el balance energético negativo mediante una dieta adecuada a las necesidades del inicio de la lactancia.

- Considerar casos previos de hipocalcemia como un criterio más de selección para el descarte de animales del sistema productivo. 
Saborío-Montero. Enfermedades Metabólicas y su impacto en la productividad del ganado lechero

\section{LITERATURA CONSULTADA}

Di Rienzo, J. A., Casanoves, F., Balzarini, M.G., González, L., Tablada, M., Robledo, C.W. 2011. InfoStat versión 2011. Grupo InfoStat, FCA, Universidad Nacional de Córdoba, Argentina. URL http://www.infostat.com.ar

Drackley, J. K. 1997. Minimizing ketosis in high producing dairy cows, pp 63-82. In TriState Dairy Nutrition Conference. Fort Wayne, Indiana.

Duffield, T. F. 2000. Subclinical ketosis in lactating Dairy cattle: Metabolic disorders of ruminants. Vet Clin. North Am. Food Anim. Pract. 16:231-253.

Epperson, W. B. 2005. Risk factors for metabolic disease. pp. 31-43. In: Tri-State Dairy Nutrition Conference. Fort Wayne.

Ferguson, J. D., Galligan, D.T., Thomsen, N. 1994. Principal descriptors of body condition score in Holstein cows. J. Dairy Sci. 77:2695-2703.

Goff, J. P. 2006. Macromineral physiology and application to the feeding of the dairy cow for prevention of milk fever and other periparturient mineral disorders. Animal Feed Science and Technology 126:237-257.

Horst, R. L. 1986. Regulation of calcium and phosphorus homeostasis in the dairy cow. J. Dairy Sci. 69: 604-616.

Iwersen, M., Falkenberg, U., Voigtsberger, R., Furderung, D., Heuwieser. 2009. Evaluation of an electronic cow-side test to detect subclinical ketosis in dairy cows. J. Dairy Sci. 92:2618-2624.

NATIONAL RESEARCH COUNCIL. 2001. Nutrient Requirements of Dairy Cattle. 7th rev. ed. National Academy Press. Washington, D.C. 381pp. 
Nutrición Animal Tropical

Noordhuizen J.P.T.M., Buurman J. 1984. Veterinary automated management and production control program for dairy farms (VAMPP). The application of MUMPS for data processing. Vet Q. 6:2, 66-72.

Oetzel, G. 2007. Herd-level ketosis-diagnosis and risk factors. American Association of Bovine Practitioners $40^{\text {th }}$ Annual Conference, September 19, 2007-Vancouver, BC, Canada pp. 67-91.

Saborío-Montero, A., Sánchez, J. Ml. 2013. Prevalencia y factores de riesgo relacionados con la cetosis clínica y subclínica tipo I y II en un hato de vacas Jersey en Costa Rica. Agronomía Costarricense 37(2):17-29.

Saborío-Montero, A., Sánchez, J. Ml. 2014. Evaluación de la condición corporal en un hato de vacas Jersey en pastoreo en la zona alta de Cartago. Variaciones durante el ciclo productivo. Agronomía Costarricense 38(1): 55-65.

Sánchez, J. M., Saborío-Montero, A. 2014 a. Prevalencia de hipocalcemia en cuatro hatos Jersey en pastoreo en Costa Rica. Agronomía Costarricense 38(2): 33-41.

Sánchez, J. M., Saborío-Montero, A. 2014 b. Hipocalcemia e hipomagnesemia en un hato de vacas Holstein, Jersey y Guernsey en pastoreo. Agronomía Costarricense 38(2): 55-65. 